\title{
NUMERICAL INVESTIGATIONS OF TWO TYPICAL UNSTEADY FLOWS IN TURBOMACHINERY USING THE MULTI-PASSAGE MODEL
}

\author{
DI ZHOU \\ College of Aerospace Engineering, Nanjing University of Aeronautics and Astronautics \\ No.29, Yudao Street, Qinhuai District, Nanjing, Jiangsu Province 210016, China \\ zhoudi0417@nuaa.edu.cn \\ ZHILIANG LU \\ College of Aerospace Engineering, Nanjing University of Aeronautics and Astronautics \\ No.29, Yudao Street, Qinhuai District, Nanjing, Jiangsu Province 210016, China \\ luzl@nuaa.edu.cn \\ TONGQING GUO \\ College of Aerospace Engineering, Nanjing University of Aeronautics and Astronautics \\ No.29, Yudao Street, Qinhuai District, Nanjing, Jiangsu Province 210016, China \\ guotq@nuaa.edu.cn

\section{ENNAN SHEN} \\ College of Aerospace Engineering, Nanjing University of Aeronautics and Astronautics \\ No.29, Yudao Street, Qinhuai District, Nanjing, Jiangsu Province 210016, China \\ shenennan@sina.cn
}

Published 22 June 2016

\begin{abstract}
In this paper, the research on two types of unsteady flow problems in turbomachinery including blade flutter and rotor-stator interaction is made by means of numerical simulation. For the former, the energy method is often used to predict the aeroelastic stability by calculating the aerodynamic work per vibration cycle. The inter-blade phase angle (IBPA) is an important parameter in computation and may have significant effects on aeroelastic behavior. For the latter, the numbers of blades in each row are usually not equal and the unsteady rotor-stator interactions could be strong. An effective way to perform multi-row calculations is the domain scaling method (DSM). These two cases share a common point that the computational domain has to be extended to multi passages (MP) considering their respective features. The present work is aimed at modeling these two issues with the developed MP model. Computational fluid dynamics (CFD) technique is applied to resolve the unsteady Reynolds-averaged Navier-Stokes (RANS) equations and simulate the flow fields. With the parallel technique, the additional time cost due to modeling more passages can be largely decreased. Results are presented on two test cases including a vibrating rotor blade and a turbine stage.
\end{abstract}

Keywords: Unsteady flow; CFD; blade flutter; rotor-stator interaction; multi-passage model.

This is an Open Access article published by World Scientific Publishing Company. It is distributed under the terms of the Creative Commons Attribution 3.0 (CC-BY) License. Further distribution of this work is permitted, provided the original work is properly cited. 


\section{Introduction}

With the rapid improvements in the performance of computer, the CFD technique has been a useful tool in the turbomachinery industry. Nowadays, traditional design methods based on steady or quasi-steady computation cannot further meet the requirements for modern and advanced aero engines. On the contrary, the research on unsteady flows has become an important and challenging task due to interior flow complexities and the subsequent computation issues. There are many inherent factors causing unsteadiness. Among them, blade flutter and rotor-stator interaction are two characteristic types drawing more and more attentions of scholars ${ }^{1-8}$.

Blade flutter is caused by interactions between aerodynamic forces and structural vibrations of the blade and could lead to blade damage and even aero-engine failure. The native and the foreign researchers have done a great deal of work on predicting blade flutter and analyzing the mechanisms. A large number of methods have also been reported. ${ }^{1,9,10}$ In engineering practice, the energy method proposed by Bendiksen ${ }^{1}$ is the most widely used approach. On the basis of the fact that the regular blade flutter manifests as a phenomenon of single-mode divergence and modes uncoupling, it assumes the blade to vibrate at some natural mode. Then the sum of the work per cycle done by unsteady aerodynamic forces is calculated. The positive value represents that the aerodynamic force damps the vibration, while on the contrary the vibration may be excited probably causing flutter. Inter-blade phase angle (IBPA) is a key parameter affecting the blade aeroelastic stability and has been studied by many authors with either experiment or numerical computation ${ }^{11,12}$.

Rotor-stator interaction is another type of unsteady problems and it usually consists of several sources of unsteadiness involving the relative motion between adjacent rows together with boundary layers, wakes and shocks. All these interactions are strongly coupled and may be further enhanced as the gap between rows is decreased. The flow losses are also produced because of these interactions. An effective type of rotor-stator interface treatment is the so-called domain scaling method (DSM) ${ }^{13}$. It models a higher number of blade passages or sometimes slightly scales the geometry to make the combination pitch distances in each row identical. The most attractive properties of DSM are the easy implementation especially for imposing periodic boundary conditions, the high robustness and the capability of capturing all time frequencies. Aube and Hirsch ${ }^{14}$ employ the DSM to investigate a 1.5 axial turbine stage at quasi-steady and fully unsteady conditions. Giboni et $\mathrm{al}^{15}$ also study the unsteady labyrinth seal leakage flow and main flow of a turbine by experiment and numerical investigation.

For both issues discussed above, multi passages are required to make the periodicity of upper and lower periodic boundaries still exists. The present CFD solver is based on our own developed code intended for unsteady turbomachinery flows. Currently, very little literature has reported three-dimensional unsteady flow experiments and publicized the measured data in detail. Therefore, two available test cases with some necessary modifications are studied, including a transonic compressor rotor with blades vibrating and an axial low-pressure turbine stage. 


\section{Numerical methods}

\subsection{Unsteady aerodynamic model}

For turbomachinery applications, it is convenient to transform the RANS equations into a rotating frame of reference. Assuming the rotation axis coincides with the x-coordinate axis, the angular velocity has the components $\omega=\left[\omega_{1}, 0,0\right]^{\mathrm{T}}$. The relationship between vectors of the absolute velocities $\boldsymbol{v}_{a}$, the relative velocities $\boldsymbol{v}_{r}$ and the convected velocities $v_{e}$ is as follows.

$$
\boldsymbol{v}_{a}=\boldsymbol{v}_{r}+\boldsymbol{v}_{e} .
$$

where $\boldsymbol{v}_{a}=[u, v, w]^{\mathrm{T}}, \boldsymbol{v}_{r}=\left[u_{r}, v_{r}, w_{r}\right]^{\mathrm{T}}$ and $\boldsymbol{v}_{e}=\boldsymbol{\omega} \times \boldsymbol{r}=\left[0,-\omega_{1} z, \omega_{1} y\right]^{\mathrm{T}}$. Considering the blade motion, an approach named as the arbitrary lagrangian eulerian (ALE) formulation is also used. Hence, the three dimensional N-S equations in the relative system for moving grids read

$$
\frac{\partial}{\partial t} \int_{\Omega} \boldsymbol{W} d \Omega+\oint_{S}\left(\boldsymbol{F}_{c}-\boldsymbol{F}_{v}\right) \cdot \boldsymbol{n} d S=\int_{\Omega} \boldsymbol{Q} d \Omega .
$$

where $\Omega$ is a moving control volume with boundary surface $S, \boldsymbol{n}$ is a unit vector. The vectors of the conservative variables $\boldsymbol{W}$, the convective fluxes $\boldsymbol{F}_{c}$, and the source term $\boldsymbol{Q}$ have the following components.

$$
\boldsymbol{W}=\left[\begin{array}{l}
\rho \\
\rho u \\
\rho v \\
\rho w \\
\rho E
\end{array}\right], \boldsymbol{F}_{c}=\left[\begin{array}{l}
\rho\left(V_{r}-V_{t}\right) \\
\rho u\left(V_{r}-V_{t}\right)+n_{x} p \\
\rho v\left(V_{r}-V_{t}\right)+n_{y} p \\
\rho w\left(V_{r}-V_{t}\right)+n_{z} p \\
\rho E\left(V_{r}-V_{t}\right)+p V
\end{array}\right], \boldsymbol{Q}=\left[\begin{array}{l}
0 \\
0 \\
\rho\left(w-w_{t}\right) \omega_{1} \\
-\rho\left(v-v_{t}\right) \omega_{1} \\
0
\end{array}\right] .
$$

where $\rho, u, v, w, p, E$ denote the density, the absolute Cartesian velocity components, the pressure and the total energy per unit mass, respectively. $V$ is the absolute contravariant velocity, $V_{r}$ is the contravariant velocity relative to the grid motion and $V_{t}$ is the contravariant velocity of the face of the control volume. The viscous fluxes which are not presented here retain the same form as in basic N-S equations. The source term is caused by the centrifugal and the Coriolis forces. It is noted that the momentum equations are expressed using the absolute velocities as dependent variables in order to treat rotor and stator rows in a unified manner.

The cell-centered based finite volume method is utilized to discretize the governing equations. The convective fluxes are evaluated by a famous approximate Riemann solver, Roe scheme. Wherein, the eigenvalues of the Jacobian of the convective fluxes are changed due to the convection motion and grid moving. A third-order MUSCL scheme with Van albada limiter function is adopted to achieve higher accuracy and keep sufficient numerical stability. The viscous fluxes are directly discretised by a secondorder central scheme. An implicit method named as LUSGS is employed with the dual 
time-stepping approach to solve unsteady equations. Because of the existence of the source term $\boldsymbol{Q}$, the diagonal operator would not be simplified to a diagonal matrix as usual $^{16}$. Given that the nonlinear time-marching method for MP model needs a large amount of calculation, the parallel technique is necessary. Here, a common parallel technology named as Message Passing Interface (MPI) is used as it could fit well with the presented method, leading to a large decrease in computational time.

A lot of inherent nonlinear factors exist in the interior flow field, such as shock boundary layer interaction, flow separation and tip leakage. All of these would become more complicated as the turbulence effects are added and raise a higher demand on numerical methods. The detached eddy simulation (DES) ${ }^{17}$ combines the good performances of RANS within the attached layer and of large eddy simulation (LES) in separated region. In the present work, either the vibration frequency or the blade passing frequency is far beyond that of turbulent fluctuating, which is suitable for DES model. Consequently, a Spalart-Allamaras (SA) one-equation ${ }^{18}$ based DES model is adopted.

$$
\frac{D \tilde{v}}{D t}=C_{\mathrm{b} 1} \tilde{\Omega} \tilde{v}-C_{\mathrm{w} 1} f_{\mathrm{w}}\left(\frac{\tilde{v}}{\tilde{d}}\right)^{2}+\frac{1}{\sigma}\left\{\nabla \cdot\left[\left(v_{\mathrm{L}}+\tilde{v}\right) \nabla \tilde{v}\right]+C_{\mathrm{b} 2}(\nabla \tilde{v})^{2}\right\} .
$$

where $v_{\mathrm{L}}$ is laminar viscosity, $\tilde{v}$ is turbulent viscosity, $\tilde{\Omega}$ is the vorticity. $\tilde{d}=\min \left(d, C_{\mathrm{DES}} \Delta\right)$ is the charactersitic turbulence length, where $d$ is the nearest distance to the wall and $\Delta$ is the grid scale. The other constants can be referred in Ref. 17 . Obviously, when $d \ll \Delta$, the DES turns to SA model while on the contrary it acts as LES.

In the MP model, the periodic boundary conditions are directly imposed. For rotating (blade, hub) and stationary walls (hub), the slipping and non-slipping boundary conditions are applied, respectively. At the inlet, the total pressure, total temperature and two flow angles are specified. By employing the non-reflecting conditions together with isentropic relations, the flow variables at the inlet can be obtained. At the outlet, the static pressure is predefined at the hub location and the radial equilibrium equation is solved to determine the outlet pressure distribution

$$
\frac{\partial p}{\partial r}=\bar{\rho} \frac{\bar{V}_{\theta}^{2}}{r}
$$

where $\bar{\rho}$ and $\bar{V}_{\theta}$ are the averaged density and tangential velocity, $r$ is the center radius. The equation actually represents the balance of the pressure and centrifugal force. The other flow variables at the outlet can be obtained by extrapolation from the interior.

\subsection{Blade vibrating problems}

An important factor in the calculation of blade flutter is the aerodynamic effects of adjacent blades. According to Lane's traveling model ${ }^{19}$, it is usually described by the IBPA, which is defined as

$$
\sigma=\frac{2 \pi N_{d}}{N_{b}}
$$


where $N_{d}$ represents the nodal diameter and $N_{b}$ is the number of blades. If $N_{d}$ is assumed to be zero, all blades vibrate in phase. For other nodal diameters, each blade will vibrate out of phase with a constant $\sigma$. And the number of passages needed to model equals to $2 \pi / \sigma$. It is well known that IBPA has significant influences on the blade aeroelastic stability. Normally, to find out the least stable $\sigma$, the unsteady flows with possible IBPAs should all be calculated.

Another key point is the treatment way of moving grids. The poor quality of dynamic mesh may have bad influence on the computed results and even cover the real physical solution. Here, the attenuation method, a simple but efficient dynamic mesh generation approach is adopted. When dealing with tip clearance, the poor mesh quality with large distortion should be prevented and meanwhile the shape of casing must be kept. Hence, the mesh points are allowed to slide along the casing surface instead of keeping them stationary as usual. Figure 1 shows an example of dynamic mesh for the tip clearance.

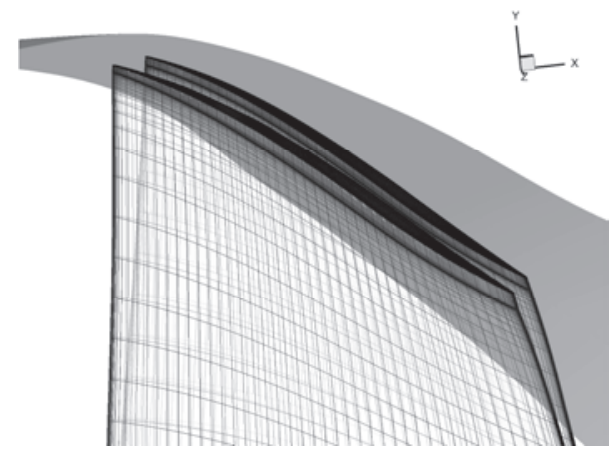

Fig. 1. Dynamic mesh for the tip clearance.

\subsection{Rotor-stator interaction}

Concerning the resonance, the numbers of blades in each row are usually not equal so that the periodic boundary conditions cannot be directly imposed. An alternative is to model the whole annulus, at the cost of enormous memory and computational time. Another efficient approach is the DSM, which is aimed at finding a periodicity existing in multi passages. For instance, if the numbers of blades are 30 and 45, the numbers of passages to model are respectively 3 and 2 in order to have the same combination pitch distances. However in some situations, it is difficult and even impossible to find an appropriate multiple. Then the geometry may be slightly scaled usually by changing the blade number. For example, if the number of blades are 29 and 45, one may still choose to model 3 and 2 passages with an error proportional to the scaling factor (here, it is 1.0345). The accuracy would be higher if the chosen scaling factor is closer to 1 , usually meaning more passages to be modeled. So one may always make balance between the requirements of accuracy and data storage together with solution time.

During time-marching process, the rotation of rotor makes the relative position to the stator changed at each time step. Because the unsteady equations are resolved in the 
relative system of each row, no grid rigid motion needs to be performed and only the interpolation data structure is modified in function of the relative position. Noticing the repeatability of this procedure, the interpolation coefficients for every time step can be calculated in advance and then stored. At the stator-rotor interface, the temporal physical variables of both rows are exchanged with each other by an area-based interpolation method to ensure the conservation of fluxes.

\section{Unsteady results for a transonic rotor}

Rotor $37^{20}$ is a transonic compressor rotor with a design speed of $17188.7 \mathrm{r} / \mathrm{min}$ and a blade number of 36. It was conducted as a steady-state aerodynamic experiment at the NASA Lewis Research Center and later retested by Suder and Celestina ${ }^{21}$ as an isolated rotor. As full details of the design parameters and measured performance are available, the experimental data was often used for a CFD test case. The computational grid is shown in Fig. 2 with an O4H-type topology for S1 stream surface and an OH-type topology for tip clearance. The total grid number is about 0.8 million with 8 cores for parallel computation.

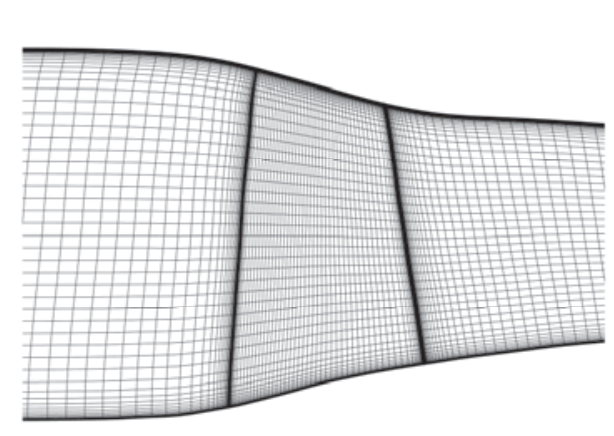

(a) S2 surface

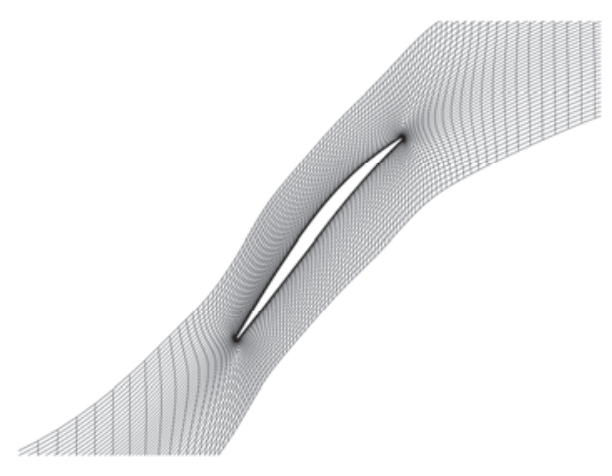

(b) S1 surface

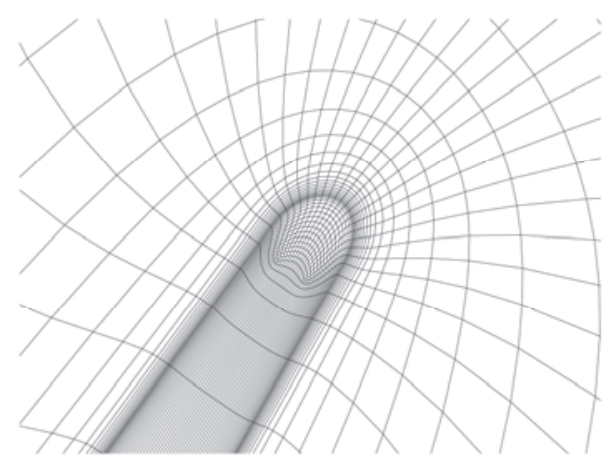

(c) Tip clearance

Fig. 2. Computational grid for Rotor 37. 
First, the steady flow characteristics of Rotor 37 are calculated and the numerical results are compared in validation of CFD code. By changing the outlet pressure specified at hub within a reasonable range, the overall performance can be obtained. Then the flow structures at the near peak condition (98\% maximum mass flow rate) are investigated in detail. Figure 3 and 4 respectively present the spanwise distributions of total pressure ratio $P_{t}$ and total temperature ratio $T_{t}$ at the outlet and the relative Mach number contour at $90 \%$ span. Also present are the experimental results.

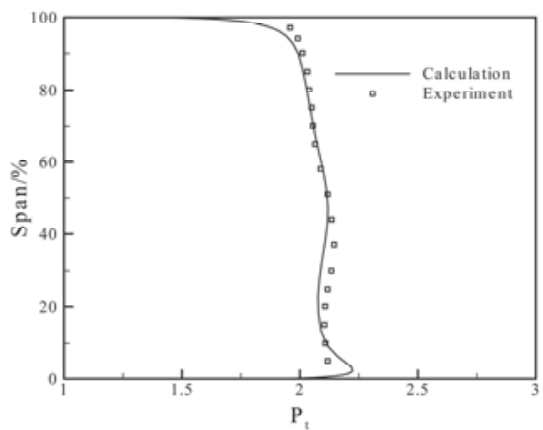

(a) Total pressure distribution

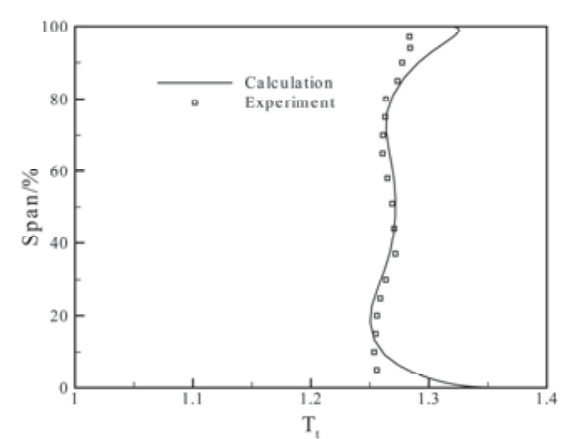

(b) Total temperature distribution

Fig. 3. Comparison of total pressure and temperature ratio distributions, near peak.
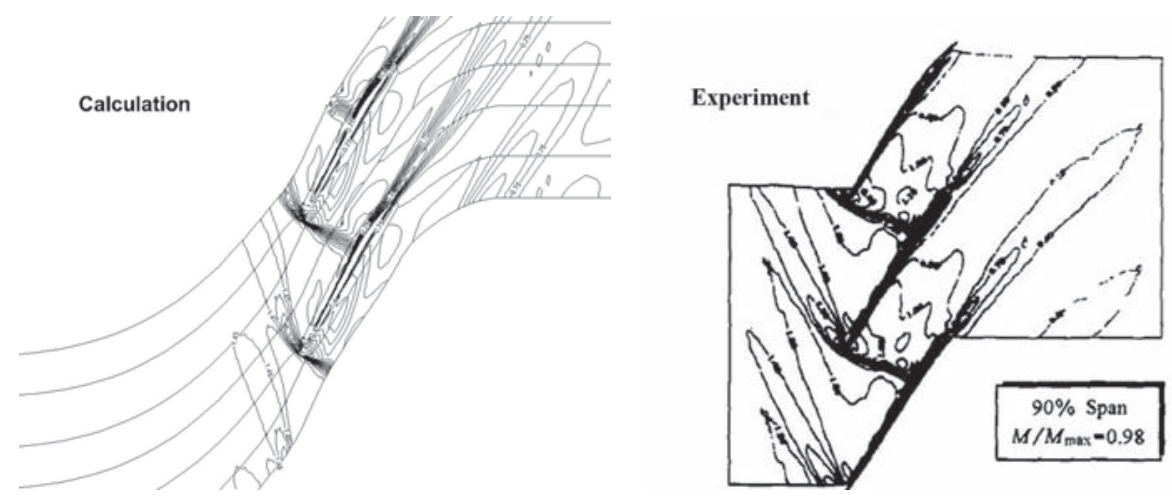

Fig. 4. Comparison of relative Mach number contours at $90 \%$ span, near peak.

Given that there is no unsteady aerodynamic or aeroelasticity test for this rotor, the blade is forced to vibrate sinusoidally at the first bending direction with a constant natural frequency. Because the original low-aspect ratio blade is rigid enough to be free of any aeroelastic problems, the first natural frequency is artificially reduced to $300 \mathrm{~Hz}$ in order to seek the potential of blade flutter. The vibration amplitude is chosen to make the maximum blade displacement to be $1 \mathrm{~mm}$ and the operating condition is selected to be near peak so that no stalled flow or other nonlinearities are included. By employing the 
energy method, four typical IBPAs $\left(0^{\circ}, \pm 90^{\circ}, 180^{\circ}\right)$ are chosen to be studied, respectively requiring 1, 2 and 4 passages. Each period is divided into 120 steps and the implicit time marching is performed in the pseudo time until the residual is fallen by one order. The aerodynamic damping computation is important allowing us to predict blade flutter, which is defined in the same form as in Ref. 21.

$$
\delta=-\frac{W_{\text {cycle }}}{\omega^{2} M h^{2}} .
$$

where $W_{\text {cycle }}$ is the total aerodynamic work per cycle, $\omega$ is the angular natural frequency, $M$ is the modal mass and $h$ is the modal amplitude. $W_{\text {cycle }}$ is calculated by

$$
W_{\text {cycle }}=\int_{0}^{T} \oint(p+\tau) \cdot \boldsymbol{v} \cdot \boldsymbol{n} d S d t .
$$

The calculated damping coefficients with different IBPAs are presented in Fig. 5. The least stable angle is found to be $0^{\circ}$, in which case the damping coefficient is negative meaning the vibration of blade is excited by the aerodynamic force and blade flutter may occur. For other three IBPAs, the blade vibrations are all damped and the most stable condition is obtained at $180^{\circ}$.

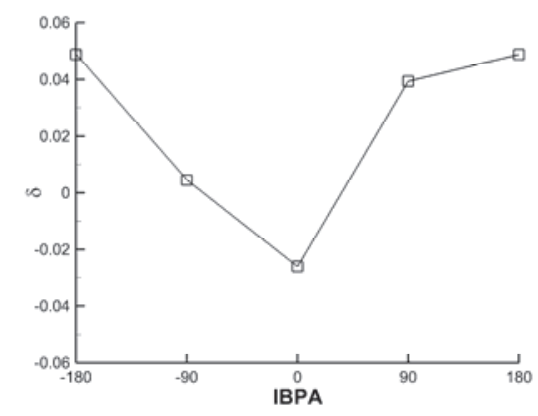

Fig. 5. Computed aerodynamic damping coefficients with IBPA.

In order to understand the effects of blade areas on the aeroelasticity, the work distributions in the blade suction and pressure surfaces for the unstable case ( $0^{\circ}$ IBPA) are presented in Fig. 6. Figure 7 also gives the time-averaged pressure distributions. From these two figures, the main findings and conclusions can be summarized into three points. Firstly, the majority region of pressure surface has very small values showing little contribution to the total work. The leading edge areas both in the suction and pressure surfaces have negative work values indicating the stability effects on the blade. Secondly, the positive-work area mainly appears at the half chord location of the upper-span sections in the suction surface. This area just corresponds to the position of strong passage-shock wave as shown in Fig. 7, which implies that in this case the shock has the instability effect and may induce blade flutter. The last point is that the areas at upper span especially near tip region have greatly more significant influences than those at lower span. This is most likely due to the high rotation speed causing the spanwise 
variety from subsonic flow to transonic and supersonic flows. As the combination of these two distinguished effects finally determine the blade aeroelastic stability, it is hard to judge whether effect is stronger in advance. It is therefore suggested to carefully consider the shock position and strength and the tip region flow in designing a blade free of flutter.

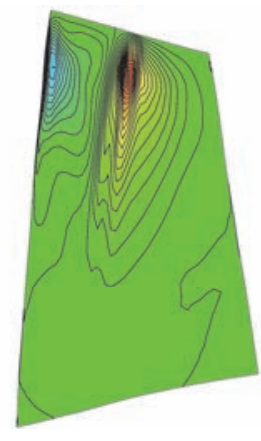

(a) Suction surface

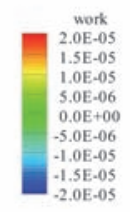

Fig. 6. Computed distributions of aerodynamic work per cycle on the blade.

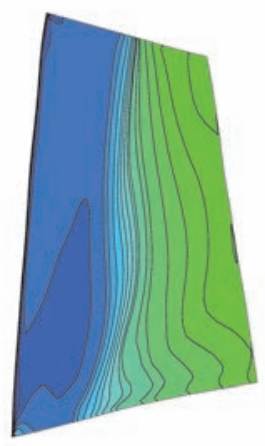

(a) Suction surface

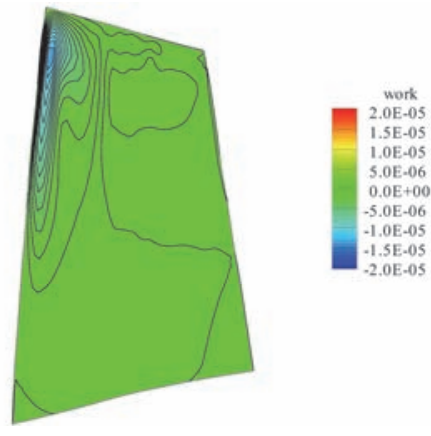

(b) Pressure surface

Fig. 7. Computed distributions of time-averaged pressure on the blade.

\section{Unsteady results for a turbine stage}

The original Aachen 1-1/2 turbine stage ${ }^{22}$ has three rows in order corresponding to the stator, the rotor and the stator and the blade numbers are respectively 36,41 and 36 . For the sake of simplicity and meanwhile not affecting our goals, the last stator is cut down and only the first two rows are simulated. The rotor has a design speed of $3500 \mathrm{r} / \mathrm{min}$ and is unshrouded with a tip clearance of $0.4 \mathrm{~mm}$. Testing conditions are characterized by an inlet total pressure of $169500 \mathrm{~Pa}$ and a total temperature of $305 \mathrm{~K}$. The inlet flow is assumed to enter the domain along the axial direction (x-axis). By using the DSM, the blade passages to be modeled for each row are approximately reduced to 4 and 5 . The 
scaling coefficient is only 1.025 and could be applicable for engineering application. The computational grid is shown in Fig. 8.

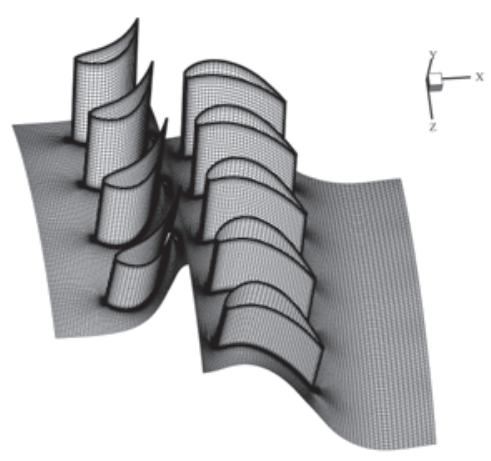

Fig. 8. Computational grid for a turbine stage.

The total grid number is about 2.3 million with 54 cores for calculation. The radial distributions of grids for the stator and the rotor row are set identical so that the interface interpolation can be seen as a one-dimensional problem. The physical time of each period is divided into 120 steps. In order to guarantee the convergence, the periodicity of the flow filed is checked. After several transition periods, a converged periodic solution is obtained as shown in Fig. 9. Besides, the calculated frequency of time histories equals to the blade-passing frequency indicating the major source of the unsteadiness is mainly from the rotor rotation.

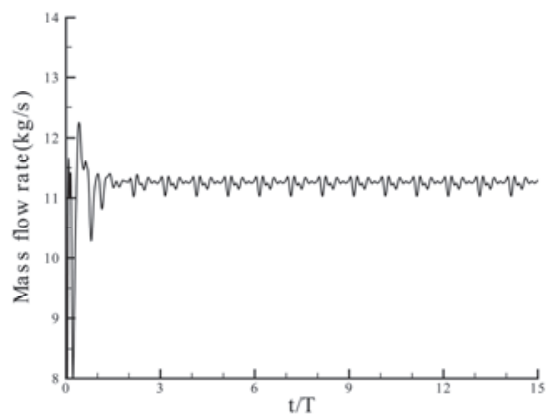

(a) Mass flow rate

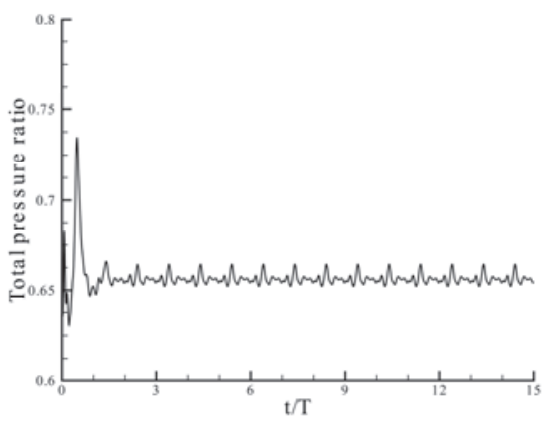

(b) Total pressure ratio

Fig. 9. Time histories of computed mass flow rate and total pressure ratio.

Then the entropy contours of the mid-span section at 4 distinct time points in a period are presented in Fig. 10. The entropy production is calculated with the inlet flow condition set as the reference state. It is obviously found that the entropy increases rapidly at the boundary layer due to the viscous effect. The wakes generated at the trailing edge spread downstream causing the so-called cutting effect to the flow filed 
structure of the rear blade row. With the joint influences of the upstream wakes and the viscosity, the entropy contours of the rotor row have become temporally and spatially complex and chaos. The overall entropy value at the outlet is now much higher than that at the inlet which represents the flow losses. Besides, at any time, the wakes continuously pass through the rotor-stator interface verifying the accuracy in space and time of the present MP model.

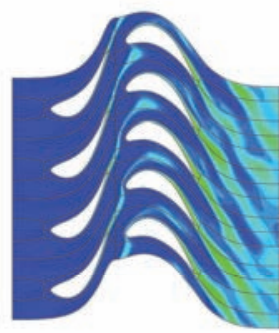

(a) $\mathrm{t}=1 / 4 \mathrm{~T}$

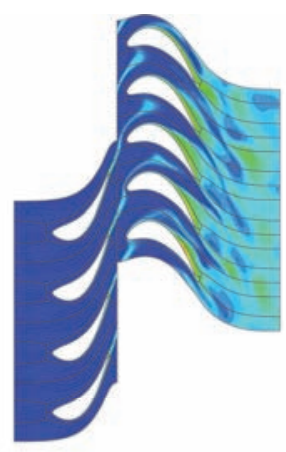

(c) $\mathrm{t}=3 / 4 \mathrm{~T}$
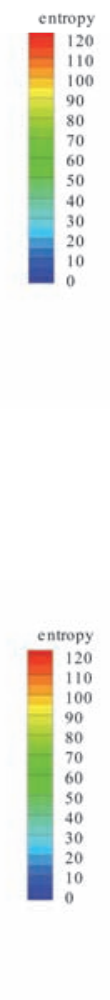

(d) $\mathrm{t}=4 / 4 \mathrm{~T}$

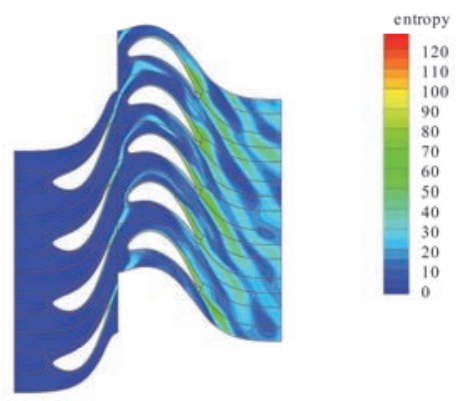

(b) $\mathrm{t}=2 / 4 \mathrm{~T}$

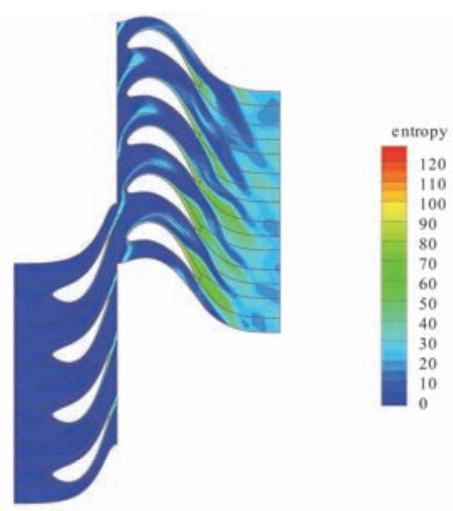

Fig. 10. Entropy contours at 4 time points in a period.

\section{Conclusions}

There are two commonly encountered unsteady flow problems in turbomachinery: blade vibration and rotor-stator interaction. Both of them need to model multi passages. Therefore an effective MP model based on CFD technique is presented and then applied in two test cases. For blade vibration problem, an efficient dynamic approach considering the treatment of tip clearance is employed. The energy method is adopted to predict the aeroelastic stability of Rotor 37 blade and briefly analyzes the mechanism of blade flutter. It is found that the blade shows the least stability at IBPA of $0^{\circ}$ and the most at $180^{\circ}$. 
The different areas in the blade have complex effects and it is observed that the passage shock would give rise to blade flutter. The sensitivities for shock position and strength need to be further researched. For rotor-stator interaction, the DSM is used with a slightly geometry modification. However, the simplification does not affect our goals. The calculated results for a turbine stage show favorable efficiency and accuracy. A converged solution is fast obtained after only 3 periods of iterations. The relevant flow characteristics of the rotor-stator problem are captured and demonstrated through the temporal entropy contours. The future work may focus on extending the method to 3 and more rows and decreasing the error introduced by geometry scaling.

\section{Acknowledgments}

This work is supported by the Priority Academic Program Development of Jiangsu Higher Education Institutions.

\section{References}

1. O. O. Bendiksen, Aeroelastic problems in turbomachines, in 31st structures, structural dynamics and materials conference, (Los Angles, California, 1990).

2. J. G. Marshall, M. Imregun, J. Fluid. Struct. 10, 237 (1996).

3. M. Vahdati, A. I. Sayma, J. G. Marshall and M. Imregun, J Propul Power. 17, 1100 (2011).

4. D. Zhou, Z. L. Lu, T. Q. Guo and E. N. Shen. Acta Aeronautica et Astronautica Sinica. 36, 1076 (2014).

5. J. J. Adamczyk. ASME Paper 85-GT-226, (1985).

6. M. B. Giles. J Propul Power. 6, 621 (1990).

7. R. Culver and F. Liu. Mixing-plane method for flutter computation in multi-stage turbomachines, in 47th AIAA Aerospace Sciences Meeting Including The New Horizons Forum and Aerospace Exposition, (Orlando, Florida, 2009).

8. M. Giovannin, M. Marconcini and A. Arnone. J Power Energy. 0, 1 (2014).

9. O. O. Bendiksen and P. P. Friedmann. J Eng Gas Turb Power. 104, 617 (1982).

10. M. Sadeghi and F. Liu. AIAA Paper 2005-18 (2005).

11. A. Bölcs and T. H. Fransson. Communication Du Laboratoire de Thermique Appliqué et de Turbomachines, (1986).

12. H. S. Im and G. C. Zha. Flutter prediction of a transonic fan with traveling wave using fully coupled fluid/structure interaction, in Proceedings of ASME Turbo Expo 2013: Turbine Technical Conference and Exposition, (San Antonio, Texas, 2013).

13. M. M. Rai. J Propul Power. 5, 305 (1989).

14. M. Aube and C. Hirsch. Numerical investigation of a 1-1/2 axial turbine stage at quasi-steady and fully unsteady conditions, in Proceedings of ASME TURBO EXPO 2001, (New Orleans, Louisiana, 2001).

15. A. Giboni and K. Wolter. Experimental and numerical investigation into the unsteady interaction of labyrinth seal leakage flow and main flow in a 1.5-stage axial turbine, in Proceedings of ASME TURBO EXPO 2004: Power for Land, Sea and Air, (Vienna, Austria, 2004).

16. J. Blazek. Computational Fluid Dynamics: Principles and Applications, 2nd edn. (Elsevier Science, Oxford, 2005).

17. S. Morton, J. Forsythe and A. Mitchell. DES and RANS simulations of delta wing vertical flows, in 40th AIAA aerospace sciences meeting and exhibition, (Reno, Nevada, 2002). 
18. P. R. Spalart and S. R. Allmaras. A one-equation turbulence model for aerodynamic flows, in 30th aerospace sciences meeting and exhibit, (Reno, Nevada. Reston, 1992).

19. F. Lane. Aeronaut J. 23, 54 (1956).

20. L. Reid and R. D. Moore. NASA TP-1337, (1978).

21. K. L. Suder and M. L. Celestina. NASA TM-106711, (1994).

22. R. E. Walraevens, H. E. Gallus and A. R. Jung. Experimental and computational study of the unsteady flow in a 1.5 stage axial turbine with emphasis on the secondary flow in the second stator, in International Gas Turbine and Aeroengine Congress \& Exhibition, (Stockhol, Sweden, 1998). 